Case report

\title{
Two cases of pediatric epithelioid sarcoma of the hand and coverage with Integra ${ }^{\circledR}$
}

\author{
Mihaela Năstasă ${ }^{*, 1}$, Vincent Casoli ${ }^{2}$ \\ ${ }^{1}$ Department of Plastic Surgery and hand Surgery, Valais Hospital, Sion, Switzerland; ${ }^{2}$ Department of \\ Plastic Surgery, CHU Bordeaux, France
}

\begin{abstract}
Epithelioid sarcoma is an uncommon soft tissue tumor of the hand characterized by high frequency misdiagnosis and high potential for local recurrence and regional lymph node metastasis. The recommended treatment is wide excision, which leaves complex tissue defects with tendon or neurovascular structure exposure requiring coverage. We present two patients who had tumor excision followed by wound coverage with dermal regeneration template and split-thickness skin graft. Integra ${ }^{\circledR}$ template represents a different approach, which provides a safe, reliable and effective means of soft tissue coverage of the hand.
\end{abstract}

Keywords: epithelioid sarcoma; pediatric; wide excision; Integra; reconstruction

\section{Introduction}

Epithelioid sarcoma is an uncommon soft tissue tumor that occurs frequently in the forearm and hand. This type of sarcoma is characterized by innocuous appearance, frequent misdiagnosing and high potential for local recurrence and regional lymph node metastasis. Presenting patients are teenage and young adults and mostly males.

The most common option for treatment of extremities sarcomas is hand-sparing surgery, consisting in wide excision and adjuvant therapy. The wide local excision supposes an increased complexity of soft-tissue and bony coverage or reconstruction. Various types of reconstructions have been described in literature: primary closure, skin grafting and pedicle or free flaps (finger fillet flap, radial forearm flap, gracilis flap) [1].

Received: August 2018; Accepted after review: August 2018; Published: September 2018.

${ }^{*}$ Corresponding author: Mihaela Năstasă, Department of Plastic Surgery and hand Surgery, Valais Hospital, Sion, Switzerland.

Email: nastasa mihaela elena@yahoo.com
In this article we report 2 patients with epithelioid sarcoma of the hand for whom the coverage was made by a dermal regeneration template (Integra®).

\section{Case report 1}

A 10-year old female was admitted to another hospital with a lesion of her right palm in December 2005. The excision of the tumor was performed under general anesthesia. Intraoperatively, the tumor was cystic, with regular borders and multiple lobules, adherent to the sheath of the flexor tendon of her right second digit.

The histopathological analysis showed a relatively well-limited lesion with epithelioid cells. The tumor cells were characterized by important cytonuclear atypia, with hyperchromatic nuclei and low mitotic activity ( $<1$ mitosis/10 high power fields). The immunohistochemical staining reveals a strong positive and diffuse expression of epithelial membrane antigen (EMA) and a focally positive expression for cytokeratin AE1/AE3 and CD34. The specimen stained negative for 
the muscle actins, desmin, protein $\mathrm{S} 100$ and CD30.

The histopathological aspect establishes the diagnosis of epithelioid sarcoma, Grade 2 of malignancy FNCLCC (Fédération Nationale des Centres de Lutte Contre le Cancer) and the patient was referred to our department.

The magnetic resonance imaging (January 2006) is imposed by the histopathological diagnosis. It shows an abnormal signal concerning the cutaneous and the subcutaneous tissue and the sheath of the flexor tendon, without bone involvement. A nodular lesion $10 / 15 \mathrm{~mm}$ high is detected just above the metacarpal phalangeal joint of the right hand thumb, on the distal part of the postoperative scar.

Wide excision was performed in February 2006 with additional margins of $2.5 \mathrm{~cm}$ circumferentially, including the muscular aponeurosis, preserving the flexor tendons and neurovascular structures. Biopsies were made in 15 different sites (Figure 1).

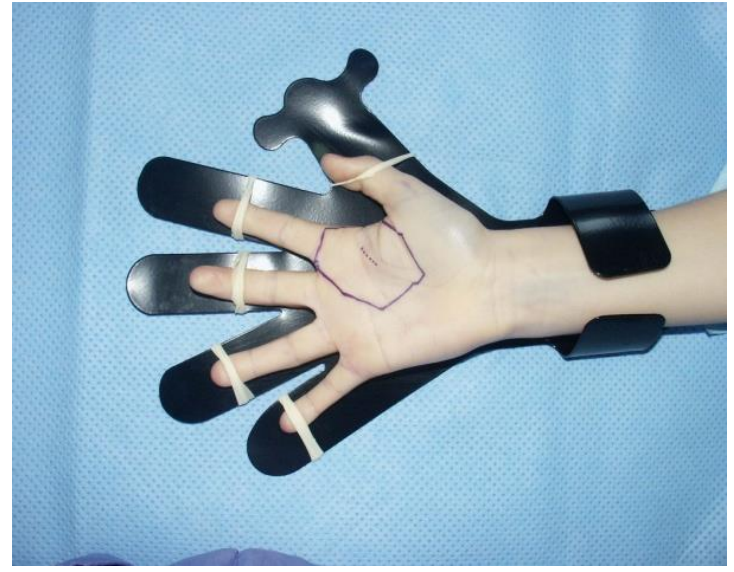

Fig. 1. Preoperative marking, additional margins of $2.5 \mathrm{~cm}$ around the postoperative scar

Excision of the tumor created a fullthickness defect with the absence of overlying fascia, subcutaneous fat, the fascia of the lumbrical muscle of the second digit and the fascia of the adductor pollicis and opponent pollicis muscle of the thumb (Figure 2).

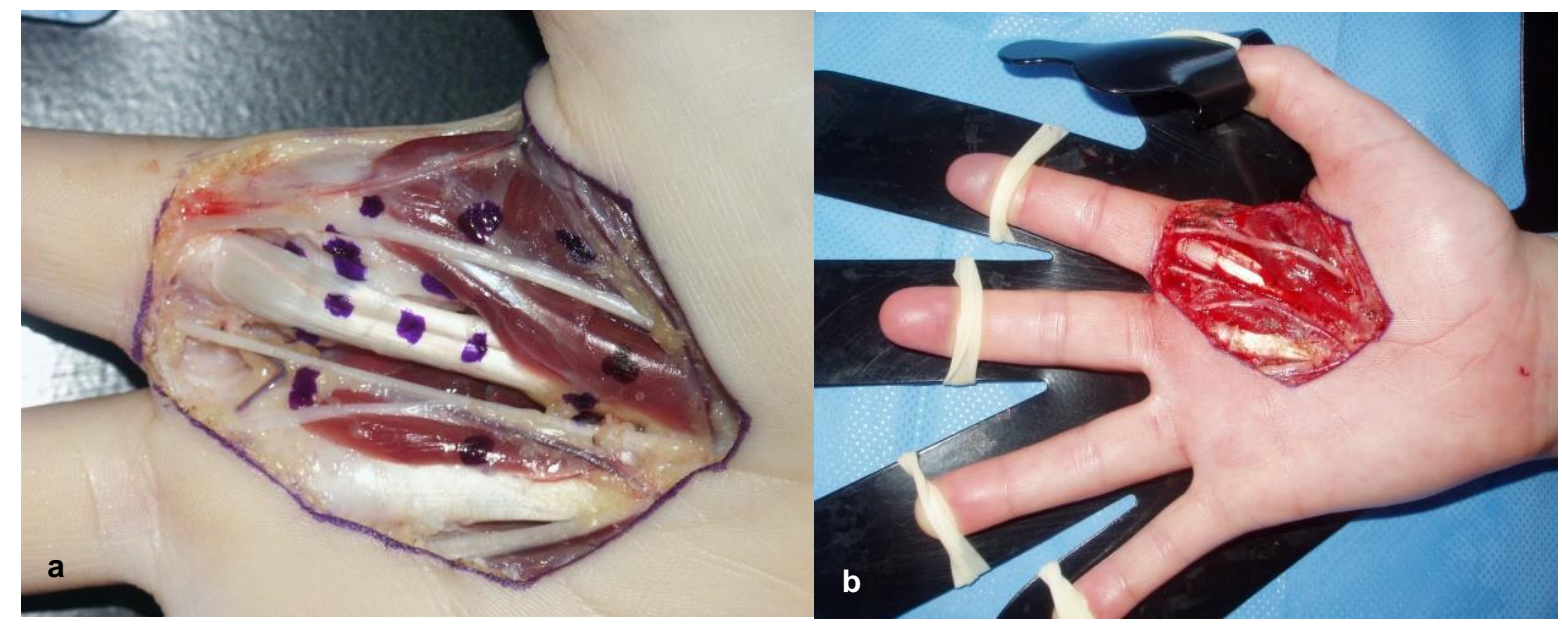

Fig. 2. Intraoperative aspect: wide excision: a) under tourniquet, the anatomical details, tendons, thenar muscles and neurovascular bundles of the index and medius; b) the defect after releasing the tourniquet

After hemostasis, the defect was covered with a dermal regeneration template (Integra $\left.{ }^{\circledR}\right)$ fixed in place with stitches and Indermil $($ glue (Figure 3). 


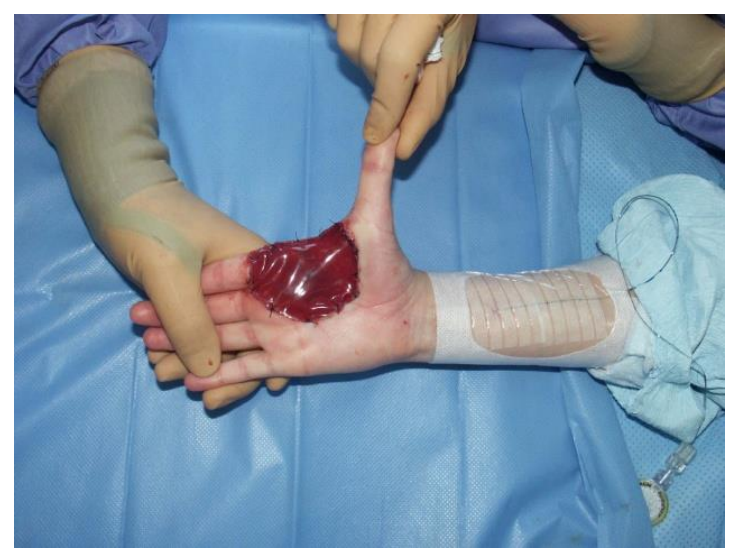

Fig. 3. Intraoperative aspect: Integra ${ }^{\circledR}$ was used to cover the defect

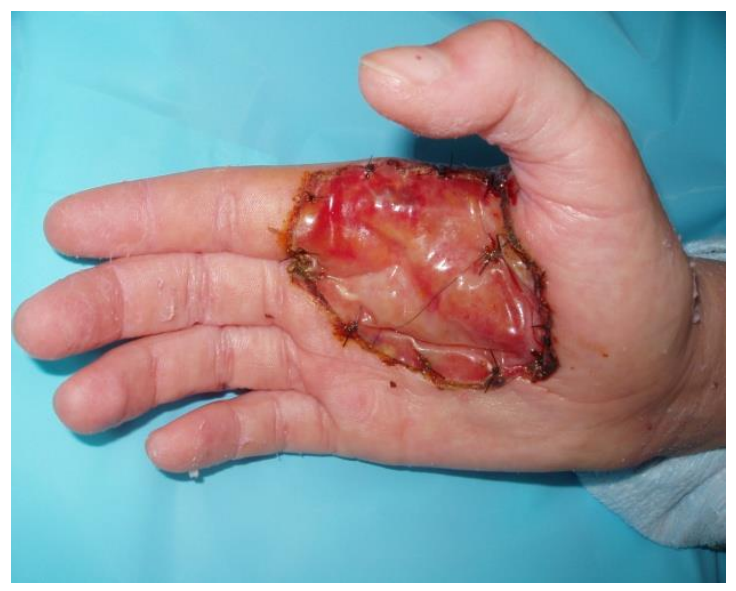

Fig. 4. Postoperative aspect of Integra ${ }^{\circledR}$ after 1 week

A split-thickness skin graft from the patient's scalp was performed 3 weeks after the initial procedure. The hand was splinted in the extension position during the entire healing period (Figure 5).

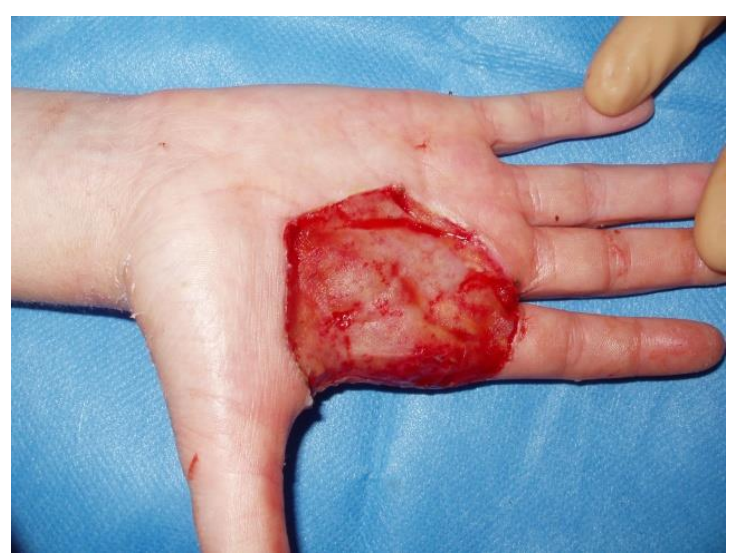

Fig. 5. Split-thickness skin graft to cover Integra ${ }^{\circledR}$
Pathological examination revealed no residual tumor on all biopsy specimens.

The wide excision was followed by radiotherapy (54 Gy), 2 months after the skin graft.

At one year follow-up, the patient presented with normal muscular force and slight stiffness in the active flexion of the proximal interphalangeal (PIP) and metacarpophalangeal joints of the right index $\left(10-15^{\circ}\right.$ total active motion TAM). No local recurrence and no regional and distant metastasis have been observed four years postoperatively.

\section{Case report 2}

A 13-year old male presented with slight swelling of the base of the palmar surface of his right fourth digit (he is left-handed) in September 2005. Clinical assessment revealed a tumor of 2-3 $\mathrm{mm}$ diameter, moderately increased, with mild pain under pressure. Granuloma was suspected and initial $10 \mathrm{~mm}$ excision was undertaken in another department in February 2006. The histological and immunohistochemical examinations established the diagnosis of epithelioid sarcoma and the patient was referred to our department.

The local magnetic resonance imaging showed a mass measuring $3 \mathrm{~mm}$, corresponding probably to the scar and the lesion appeared totally excised. Clinically, the patient was in good general health with a transverse scar on the base of the palmar right fourth digit. $\mathrm{He}$ had no palpable lymphadenopathy and all tests were within the standard limits.

Secondary surgery was performed in March 2006, consisting in wide local excision (Figure 6) sparing the neurovascular bundle laterally and the digit sheath medially, with 14 biopsy specimens. The additional margins are the distal palmar flexion crease, palmar flexion PIP crease and laterally at the dorso-lateral junction. The total excision of the digit sheath of the flexor tendon required the reconstruction of the A2 pulley. The palmaris longus was absent and the reconstruction of the pulley was made with the extensor indicis proprius with the technique of "double lacage". 


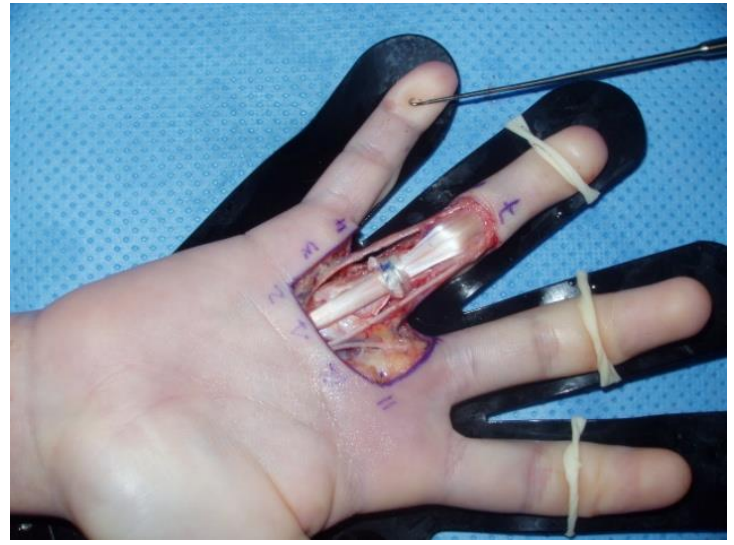

Fig. 6. Intraoperative aspect: wide local excision, neurovascular bundles intact and the pulley reconstructed.
The defect was covered with dermal regeneration template (Integra $\AA$ ), which was set in place with stitches and Indermil $\AA^{\circ}$ glue; Integra ${ }^{\circledR}$ was fully revascularized by day 21 (Figure 7).

A split-thickness skin graft from the patient's scalp was performed 3 weeks after the initial procedure. The right fourth digit was splinted in extension position during graft intaking period (Figure 8).

Pathological examination revealed no residual tumor in all biopsy specimens. The second surgery was followed by radiotherapy (57.40 Gy) 2 months after the skin graft.
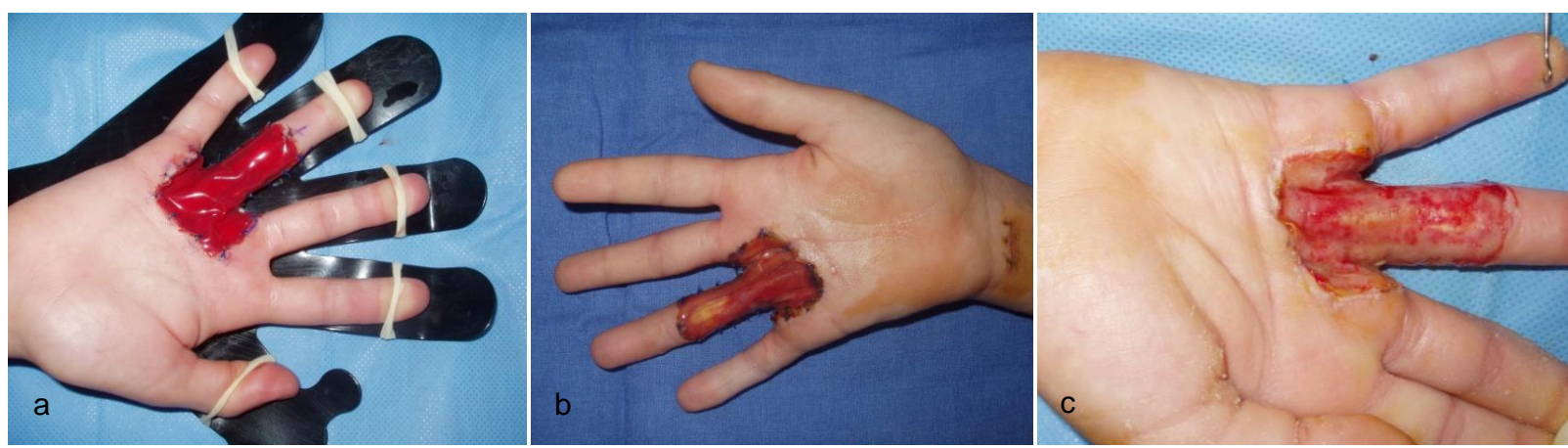

Fig. 7. Integra $₫$ to cover the wound bed: a) intraoperatively; b) at 3 weeks; c) at 3 weeks after removing the silicone component

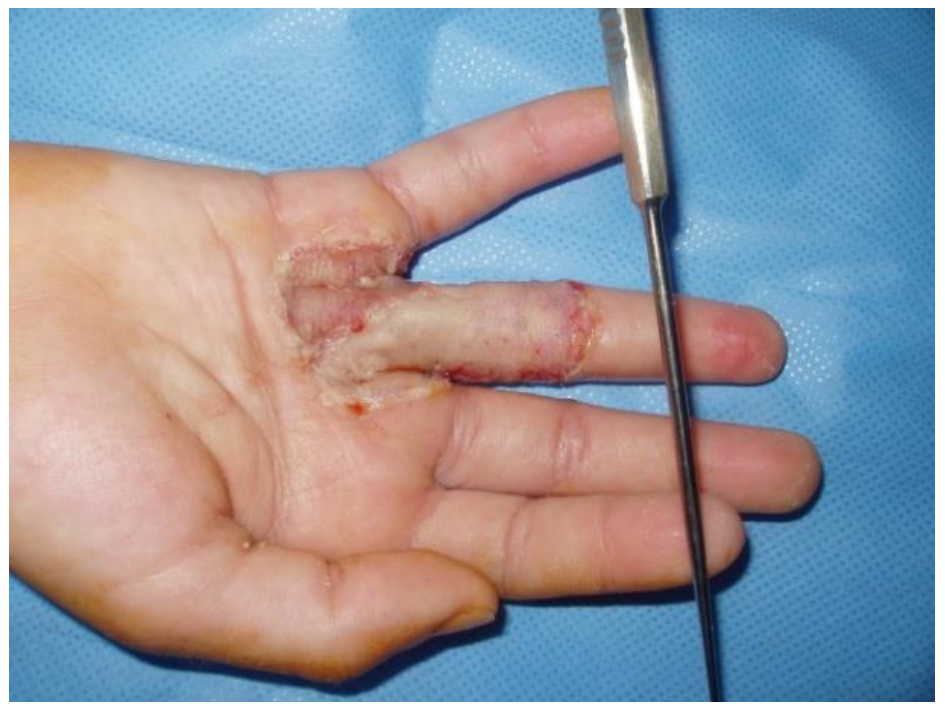

Fig. 8. Split-thickness skin graft applied on top of the dermal template of Integra®

At one year follow-up, after surgery and radiotherapy, the patient presented stiffness in the active flexion of the proximal and distal interphalangeal joints of the fourth right digit. Slight stiffness in the flexion of PIP joint of the fourth right digit and a $40^{\circ}$ flexion of the distal interphalangeal joint of the fourth right digit was present four years after treatment (Figure 9). 


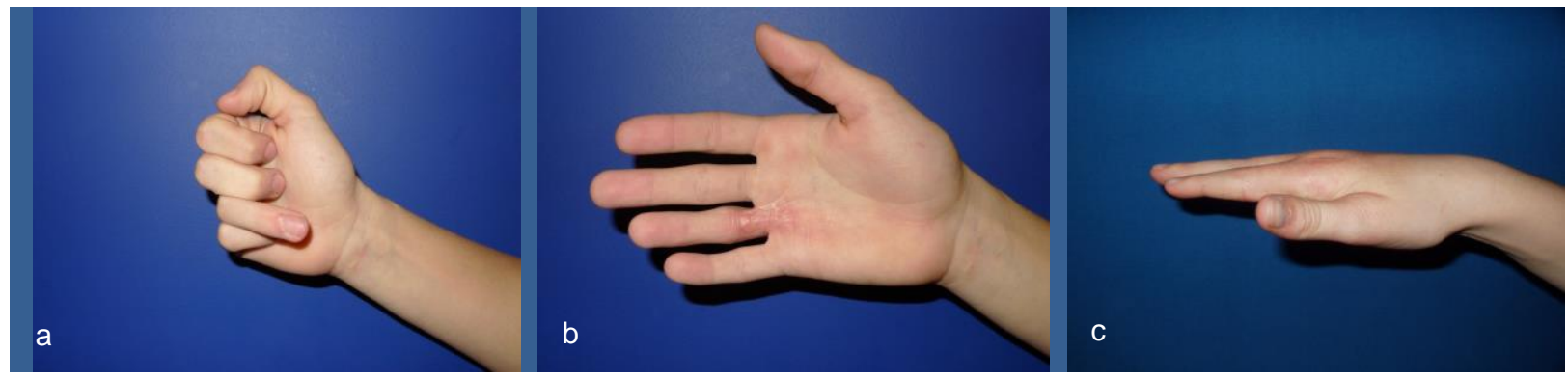

Fig. 9. Four years after treatment: a. palmar view in flexion: slight stiffness in the flexion of PIP joint and a flexion $40^{\circ}$ of the distal interphalangeal joint of the fourth right digit; b. palmar view in extension ; c. lateral view in extension

\section{Discussions}

The epithelioid sarcoma as a distinct entity was initially described by F.M. Enzinger in 1970, who published a larger series in 1985 [2-4]. This tumor is the most common soft tissue sarcoma, mostly in distal upper extremities, mainly affecting young adults, with a male predominance. A history of trauma has been reported in $20 \%$ of the cases [3]. Other authors reported the tumor being second to malignant fibrous histiocytoma [5-7] or synovial sarcoma [8, 9]. In general, soft tissue sarcoma of the hand has an incidence of $3-5 \%$ of all extremities sarcomas [6, 10] and an incidence of $7 \%$ of malignant neoplasms seen in children [11].

The tumor is remarkable for being clinically and histologically misdiagnosed and its high potential for local recurrence and regional lymph node metastasis. Clinically, the epithelioid sarcoma presents as a slowgrowing, painless and firm nodule with irregular or indistinct infiltrating margins, which is palpable in the deep soft tissues, subcutis or dermis. In evolution, ulceration is frequently seen and, eventually, multiple nodules become palpable [10, 12-16].

Due to its anodyne aspect in the initial stages of the disease and the clinical similarity to an inflammatory process, the epithelioid sarcoma is often mistaken for another lesion. The clinical differential diagnosis includes a variety of other benign and malignant conditions of the hand, including mycobacterial or deep fungal infection [12], Dupuytren's nodules, rheumatoid nodules, callus, posttraumatic changes, granulomas, fibromyoma or fibrosis, inflammatory change, dermatofibroma, sweat gland or other skin tumors, and malignant tumors including synovial sarcoma and squamous cell carcinoma [10, 12, 13, 17]. The two cases we report were initially clinically diagnosed as a cystic lesion and a granuloma, respectively.

Histologically, the epithelioid sarcoma is often confused with a granulomatous process because of the characteristic nodular or multinodular growth pattern often associated with central necrosis and oval or polygonal cells with abundant eosinophilic cytoplasm [3, $10,12]$. The characteristic epithelioid cells, the pleomorphism and higher mitotic rate may be seen only in advanced stages or in recurrent tumors. Immunohistochemical tests are required for the differentiation and for the exclusion of the granulomatous conditions.

In spite of its slow-growing character, the tumor presents a high local recurrence rate unless it is adequately treated and metastases may occur years after the diagnosis and treatment. The epithelioid sarcoma is characterized by tendency to spread in the adjacent anatomic areas, along the course of tendons, fascial planes, or through blood and lymphatic vessels. The most common sites of metastasis are the lungs and regional lymph nodes [13, 17]. The patients must be constantly examined for the epitrochlear and axillary lymphadenopathy. The X-ray and CT scan of the chest completes the investigation. The local recurrence rate of $77 \%$ [2], the frequency of regional lymph node metastasis between 22 and 45\% and the distant metastatic disease rate of $45 \%$ [3] have been reported in literature.

The prognosis of the epithelioid sarcoma is dependent on the size, type and depth of 
the tumor in relation with the deep fascia and on local recurrence and metastasis. In terms of disease prognosis, patients with tumors located on distal limbs have a better prognosis than those with proximal limb or axial tumors. The epithelioid sarcomas diagnosed and treated at earlier ages and also in females behave more favorably. The epithelioid sarcomas diagnosed and treated at earlier ages and also in females behave more favorably $[2,14,15]$.

The present cases did not show local spread, involvement of the regional lymph nodes or the distant metastasis. The optimal treatment of epithelioid sarcoma involves interdisciplinary cooperation between surgical oncologists, plastic, orthopedic and vascular surgeons and specialists in irradiation therapy to complete the surgical treatment. The team should strive for local and regional control of the disease. Nonetheless, from a functional point of view, the purpose is to preserve the limb anatomy along with the function $[1,18]$.

The recommended treatment of the epithelioid sarcoma is the wide local excision or amputation in combination with radiotherapy and/or chemotherapy. For some authors, amputation is the procedure of choice if the tumor involves bone or nerves [19] or digital amputation and ray amputation if the tumor involves the fingers [20].

It has been reported that radiotherapy of the hand is poorly tolerated with the development of acute edema, persistent pain, stiffness, fibrosis and resulting loss of function. Radiotherapy of the hand is associated with an increased risk of long term toxicity and poor outcome [19, 21]. In spite of its side effects, the adjuvant radiation therapy has been shown to improve local control of soft tissue sarcoma of the extremities [6], but it has no proved role in decreasing the risk of local recurrence when excision margins were not clear [26].

The wide local excision results in small to large or complex defects with the exposure or loss of important anatomical elements. The size, composition and the anatomical location of the defect are the determinant factors of the coverage or reconstructive options, following the "reconstructive ladder". Occasionally, in complicated cases such as previously irradiated patients, the "reconstructive elevator" must be employed to ensure wound healing [18]. The different methods vary from primary closure, skin grafting to local, regional and free flaps: finger fillet flap, radial forearm flaps, gracilis flap [1,7], and fascial temporal flap. In one case of soft tissue sarcoma, the reconstruction with an iliac crest autograft and a dorsalis pedis free flap including tendons has been reported [22]. In advanced malignant tumor of the wrist, even resection-replantation was performed [9].

In our patients, the lesions were initially considered benign and treated by simple excision. After the diagnosis of epithelioid sarcoma established by the histological and immunochemical examinations, local magnetic resonance imaging reevaluated the excision area. Wide re-excision has been reported in literature to achieve microscopically negative surgical margins, thus it is an effective method for local disease control in selected patients who present after unplanned excision of the primary tumor [23].

In both cases, the wide excision left complex tissue defects with exposed tendons and neurovascular structures that required coverage. Different methods of coverage were taken into account.

Split-thickness skin graft was excluded because of the exposure of important elements and the risk of ulceration after radiotherapy. A local and regional flap can be a solution, but pose some difficulty in postoperative surveillance. Vascularized fascial free flaps such as the tensor fascia lata flap and the temporo-parietal flap can have good postoperative results. In general, the free flaps imply a longer operating time because of the dissection of the donor site vessels, the microsurgical sutures, the morbidity of the donor site and the larger surgical team and the short-term flap surveillance.

Our choice for coverage was the dermal regeneration template (Integra $\AA$ ) followed by a split-thickness skin graft. This type of reconstruction allowed shorter operating time, no aesthetic deformity compared with the donor site of the flap harvesting, but two different surgical interventions.

In literature, one case initially misdiagnosed as acral myxoinflammatory fibroblastic sarcoma, turned out to be nodular 
fasciitis of the tendon sheath. The patient was treated by wide excision followed by the stacking of a dermal regeneration template and split-thickness skin graft [24].

Integra ${ }^{\circledR}$ template is a bilayer construct with a bottom layer functioning as a dermal substitute and a selective membrane on top, with functions similar to the epidermal layer. The dermal replacement part, made of a threedimensional porous matrix of cross-linked bovine collagen and chondroitin-6-sulfate, promotes cellular ingrowth. The superficial layer consists of a thin polysiloxane (silicone) layer that controls the moisture loss from the wound bed and mechanically protects the wound.

The immediate advantages of Integra ${ }^{\circledR}$ are: the immediate physiological wound closure that allows healing to commence and facilities the early recovery, no immunological rejection and no need to use temporary covering. The long term benefits of the dermal regeneration template are: permanent autologous dermal tissue regeneration, the absent or minimal hypertrophic scarring, reduced extent of wound contracture and well to excellent joint motion. The reconstruction with Integra $\AA$ restores the skin elasticity and mechanical stability and improved cosmetic appearance [25].

The dermal regeneration template was nicely incorporated in two cases and excellent

\section{References}

1. Talbot SG, Mehrara BJ, Disa JJ, et al. Softtissue coverage of the hand following sarcoma resection. Plast Reconstr Surg 2008; 121(2):534-543.

2. Chase DR, Enzinger FM. Epithelioid sarcoma. Diagnosis, prognostic indicators and treatment. Am J Surg Pathol 1985; 9(4):241-263.

3. Enzinger FM. Epithelioid sarcoma. A sarcoma simulating a granuloma or a carcinoma. Cancer 1970; 26(5):1029-1041.

4. Fisher C. Epithelioid sarcoma of Enzinger. $A d v$ Anat Pathol 2006; 13(3):114-121.

5. Gustafson P, Arner M. Soft tissue sarcoma of the upper extremity: descriptive data and outcome in a population-based series of 108 adult patients. J Hand Surg Am 1999; 24(4):668-674. wound healing and coverage were achieved. The coverage permits a quasi-normal active flexion. The first patient presents only slight stiffness in the flexion of the PIP and metacarpo-phalangeal joints of the right second digit $\left(10-15^{\circ}\right)$. The second patient presents slight stiffness in the flexion of PIP joint of the fourth right digit and a $40^{\circ}$ flexion of the distal interphalangeal joint of the fourth right digit.

\section{Conclusions}

These two case reports suggest a different but successful approach of the coverage after the wide excision of the soft tissue sarcoma of the hand. Integra $\AA$ - the dermal regeneration template - provides a safe, reliable and effective means of the soft tissue coverage, function and appearance of the hand, even in irradiated cases.

\section{Consent}

Written informed consent was obtained from the patient for publication of this case report.

\section{Competing interests}

The authors declare that they have no competing interests.
6. McPhee M, McGrath BE, Zhang P, Driscoll D, Gibbs J, Peimer C. Soft tissue sarcoma of the hand. J Hand Surg Am 1999; 24(5):1001-1007.

7. Muramatsu K, Ihara K, Doi K, Hashimoto T, Taguchi T. Sarcoma in the forearm and hand: clinical outcomes and microsurgical reconstruction for limb salvage. Ann Plast Surg 2009; 62(1):28-33.

8. Brien EW, Terek RM, Geer RJ, Caldwell G, Brennan MF, Healey JH. Treatment of softtissue sarcomas of the hand. J Bone Joint Surg Am 1995; 77(4):564-571.

9. Kawai $A$, Hasizume $H$, Sugihara $S$, Morimoto $\mathrm{Y}$, Inoue $\mathrm{H}$. Treatment of bone and soft tissue sarcomas of the hand and wrist. Int Orthop 2002; 26(1):26-30. 
10. Sobanko JF, Dagum AB, Davis IC, Kriegel DA. Soft tissue tumors of the hand. 2. Malignant. Dermatol Surg 2007; 33(7):771-785.

11. Gross E, Rao BN, Pappo AS, et al. Soft tissue sarcoma of the hand in children: clinical outcome and management. $J$ Pediatr Surg 1997; 32(5):698-702.

12. Burgos $A M$, Chávez JG, Sánchez JL, Sánchez NP. Epithelioid sarcoma: a diagnostic and surgical challenge. Dermatol Surg 2009; 35(4):687-691.

13. Green D, Hotchkiss R, Pederson W, Wolf S. Green's Operative Hand Surgery, $5^{\text {th }}$ Edition. Philadelphia: Churchill, 2005.

14. Güngör E, Aköz T, Karakayali $G$, Artüz F, Erdoğan B, Alli N. Epithelioid sarcoma: a confusing rare tumor on the hand. Plast Reconstr Surg 1998; 102(3):925-926.

15. Pai KK, Pai SB, Sripathi H; Pranab, Rao P. Epithelioid sarcoma: a diagnostic challenge. Indian J Dermatol Venereol Leprol 2006; 72(6):446-448.

16. Prat J, Woodruff JM, Marcove RC. Epithelioid sarcoma. An analysis of 22 cases indicating the prognostic significance of the vascular invasion and regional lymph node metastasis. Cancer 41(4):1472-1487.

17. Mathes SJ, Rod Hentz V. Plastic Surgery, $2^{\text {nd }}$ Edition.Philadelphia: Saunders, 2005.

18. Throne $\mathrm{CH}$, Bartlett SP, Beasley RW, Aston SJ, Gurtner GC, Spear SL. Grabb and Smith's
Plastic Surgery, $6^{\text {th }}$ Edition. Philadelphia: Lippincott Williams \& Wilkins, 2007.

19. Owens JC, Shiu MH, Smith R, Hajdu SI. Soft tissue sarcomas of the hand and foot. Cancer. 1985; 55(9):2010-2018.

20. Gürkan V, Ozger H. Sarcomas of the hand. Acta Orthop Traumatol Turc 2007; 41(4):286290.

21. Pradhan A, Cheung YC, Grimer RJ, et al. Softtissue sarcomas of the hand: oncological outcome and prognostic factors. J Bone Joint Surg Br 2008; 90(2):209-214.

22. Osaka S, Hoshi M, Sano S, Nozaki M, Yamamoto M. Description of new composite tissue transfer for salvage of a complex hand defect. Clin Orthop Relat Res 1996; (328):9193.

23. Lin PP, Guzel VB, Pisters PW, et al. Surgical management of soft tissue sarcomas of the hand and foot. Cancer 2002; 95(4):852-861.

24. Carothers JT, Brigman BE, Lawson RD, Rizzo $M$. Stacking of a dermal regeneration template for reconstruction of a soft-tissue defect after tumor excision from the palm of the hand: a case report. $J$ Hand Surg Am 2005; 30(6):1322-1326.

25. Integra® dermal regeneration template. Information for Patients and their Families [https://www.accessdata.fda.gov/cdrh docs/pdf /P900033S008d.pdf available at 09.23.2018]. 